\title{
Prospective Observational Study to Determine Kinetics of Procalcitonin in Hospitalized Children Receiving Antibiotic Therapy for Non-Critical Acute Bacterial Infections
}

\author{
Sophie E. Katz (1) · Jennifer Crook - Rendie McHenry • \\ Andras Szeles · Natasha Halasa $\cdot$ Ritu Banerjee
}

Received: September 7, 2020 / Accepted: October 8, 2020 / Published online: October 16, 2020

(C) The Author(s) 2020

\section{ABSTRACT}

Introduction: The kinetics of procalcitonin in pediatric patients with non-critical acute bacterial infections receiving appropriate antibiotic therapy are not well described.

Methods: We performed a single-center, prospective observational pilot study of children admitted to a tertiary care children's hospital who were receiving antibiotics for treatment of a non-critical acute bacterial infection, and we prospectively measured serial procalcitonin levels daily for 4 days during hospitalization.

Results: Among the 46 children with baseline procalcitonin levels enrolled in the study, procalcitonin kinetics followed a half-life of approximately $24 \mathrm{~h}$ in most patients. Procalcitonin declined faster than C-reactive protein over the first $48 \mathrm{~h}$ of appropriate antibiotic treatment. There was variation in biomarker levels among participants with the same

S. E. Katz $(\varangle)$ - R. McHenry · N. Halasa · R. Banerjee Division of Pediatric Infectious Diseases, Vanderbilt University Medical Center, Nashville, TN, USA

e-mail: sophie.e.katz@vumc.org

J. Crook

College of Medicine, The University of Tennessee

Health Science Center, Memphis, TN, USA

A. Szeles

Department of Medicine, University of Rochester,

Rochester, NY, USA infection type, especially in participants with bacteremia, musculoskeletal infection and skin/soft tissue infection.

Conclusion: Utility of procalcitonin as a biomarker to follow every $24-48 \mathrm{~h}$ in non-critically ill children receiving antibiotic therapy for bacterial infections as an objective measure of clinical improvement is promising.

Keywords: Kinetics; Pediatric; Procalcitonin

\section{Key Summary Points}

Among hospitalized children with serious bacterial infections, PCT declined faster than CRP over the first $48 \mathrm{~h}$ of appropriate antibiotic treatment.

Proclacitonin kinetics followed a half-life of approximately $24 \mathrm{~h}$ in most pediatric patients.

There was variation in biomarker levels among participants with the same infection type.

The inclusion of single or serial procalcitonin values could strengthen pediatric prediction scoring systems. 


\section{DIGITAL FEATURES}

This article is published with digital features, including a summary slide to facilitate understanding of the article. To view digital features for this article go to https://doi.org/10.6084/ m9.figshare.13061579.

\section{INTRODUCTION}

Procalcitonin (PCT) has been investigated as a biomarker for prediction of serious bacterial infection in febrile infants and critically ill adult patients with pneumonia and sepsis [1-8]. In adults with microbiologically confirmed bacterial infections, PCT levels have been shown to rise early in infection (2-4 h after a bacterial stimulus), peak after $12-24 \mathrm{~h}$ and decrease with initiation of effective antibiotic therapy with a half-life of around $24 \mathrm{~h}[9,10]$. In the event that PCT is measured too early in the disease course, it can be falsely low and should be repeated in patients with other signs and symptoms consistent with bacterial infection. In adults, clinical syndromes with the most data supporting use of PCT include sepsis, pneumonia and chronic obstructive pulmonary disease [11, 12]. The 2019 Infectious Diseases Society of America (IDSA) Community Acquired Pneumonia guidelines suggest that PCT can be used to decrease duration of therapy for pneumonia but should not be used to determine need to initiate antibiotics in someone with clinical suspicion of pneumonia [12]. Similarly, the 2016 Surviving Sepsis Campaign guidelines recommend the use of PCT for antibiotic de-escalation but not for determining need to initiate antibiotics [13]. There are fewer data to support the use of PCT use in meningitis or in infectious complications of trauma, burns and pancreatitis [11]. Recent studies have evaluated the role of PCT in identifying bacterial infection in children with pneumonia, musculoskeletal infection, central line and fever and urinary tract infections, with varying utility of PCT to predict bacterial vs. non-bacterial infection [14-18].

Causes of falsely elevated procalcitonin levels have been described and include massive stress (i.e., severe trauma, surgery, cardiac shock, burns or cardiopulmonary bypass), prolonged severe cardiogenic shock or organ perfusion abnormalities, malaria and some fungal infections, systemic vasculitis and acute graft vs. host disease, treatment with agents that stimulate cytokines (i.e., OKT3, anti-lymphocyte globulins, alemtuzumab, IL-2 or granulocyte transfusion), end-stage renal disease or peritoneal dialysis and paraneoplastic syndromes due to medullary thyroid and small cell lung cancer [19].

Although clinicians with access to PCT use serial measurements to determine clinical improvement and response to antibiotics, data describing the kinetics of PCT in children are lacking [20-23]. To determine whether PCT can be used as a surrogate of clinical response to help guide antibiotic de-escalation in non-critically ill children, we evaluated the kinetics of PCT among children with non-critical acute bacterial infections who are receiving effective antibiotic therapy.

\section{METHODS}

\section{Study Design}

We performed a single-center, prospective observational pilot study of children admitted to a tertiary care children's hospital who were receiving antibiotics for treatment of a noncritical acute bacterial infection.

\section{Study Setting and Population}

Children between ages 1 week to $\leq 19$ years who were hospitalized at Vanderbilt Children's Hospital with an acute, non-critical bacterial infection from March 2018 through April 2019 were eligible for study inclusion. Each day, participants with initiation of antibiotics in the prior $24 \mathrm{~h}$ were identified through the electronic medical record. Acute, non-critical bacterial infection was defined as culture-proven bacteremia or urinary tract infection, or nonculture confirmed diagnoses (based on clinical and/or radiographic findings), which included antibiotic pre-treated urinary tract infection, 
retropharyngeal abscess, peritonsillar abscess, orbital cellulitis, septic arthritis and osteomyelitis. Exclusion criteria included children younger than 7 days of age or children admitted to the neonatal intensive care unit. This study was approved by the Vanderbilt University institutional review board. The study was performed in accordance with the Helsinki Declaration of 1964 and its later amendments. All subjects provided informed consent to participate in the study.

\section{Biomarker Measurements}

The first plasma sample for PCT testing was collected as leftover blood from routine laboratory testing obtained in a lithium heparin gel tube at the time of hospital presentation and prior to antibiotic administration. Serial plasma samples were then prospectively collected daily for 4 days or until hospital discharge. Samples were refrigerated within $3 \mathrm{~h}$ of collection, centrifuged and separated into two aliquots within $72 \mathrm{~h}$ of collection, and the plasma was then frozen at $-80^{\circ} \mathrm{C}$, until PCT testing was performed using a bioMérieux Vidas ${ }^{\circledR} 3$ platform. Samples underwent one freeze-thaw cycle prior to testing. Results of PCT testing were not shared with providers and were not used for clinical care. Results of C-reactive protein (CRP), white blood cell count, other laboratory tests obtained for routine clinical care and blood, urine and cerebrospinal fluid bacterial, viral and fungal cultures were abstracted from the electronic medical record. Percent change from baseline was calculated for each participant's biomarker levels each day by subtracting their PCT or CRP level from baseline, divided by the baseline biomarker level and multiplying by 100. For the subset of 20 patients who had all five PCT levels drawn, we additionally calculated daily rate of change.

\section{Clinical Outcomes}

Clinical and demographic characteristics at enrollment and during the study period were obtained through electronic medical record review and included diagnosis, age, sex, race/ ethnicity, underlying chronic medical diagnoses, hospital unit on admission and surgical procedure. An antibiotic was considered effective if the organism was susceptible to it or when a participant demonstrated clinical improvement while receiving antibiotic therapy in the absence of susceptibility data. An infectious syndrome was characterized as one of the following: bacteremia, brain abscess, meningitis, osteomyelitis or myositis without bacteremia, osteomyelitis or myositis with bacteremia, skin/soft tissue infection without bacteremia or urinary tract infection.

\section{Statistical Analysis}

Paired $t$-tests and Wilcoxon signed-rank tests were used to compare mean/median PCT and CRP levels and change from baseline. All analyses were performed using Stata version 15.1 for Mac.

\section{RESULTS}

A total of 50 children were enrolled during the 13-month study period. After enrollment, four children had no leftover blood for initial PCT sample testing and were excluded from further data analysis, leaving a total sample size of 46 children. Demographic data are shown in Table 1, with a mean age of 6.9 years (range 1.04 weeks to 18.2 years), and 25 (54.4\%) participants were female. All participants were started on effective antibiotic therapy immediately after baseline labs were obtained. The majority $(38 / 46,83 \%)$ of participants had culture-confirmed bacterial infection. Aside from surgery, none of the patients had or were exposed to any potential causes of falsely elevated PCT including massive stress, shock, systemic vasculitis, treatment that stimulates cytokines, end-stage renal disease, peritoneal dialysis or paraneoplastic syndromes. Median baseline PCT levels were similar for participants with gram-positive infection $(n=24)$ and gramnegative infection $(n=12)$ at 1.22 and $1.25 \mu \mathrm{g} / \mathrm{l}$, respectively $(p=0.8)$. When comparing mean baseline PCT levels by clinical syndrome, meningitis $\quad(55.4 \mu \mathrm{g} / \mathrm{l}, \quad n=2) \quad$ and 
Table 1 Cohort demographics

\begin{tabular}{|c|c|c|c|c|}
\hline & $\begin{array}{l}\text { All subjects } \\
(n=46)\end{array}$ & $\begin{array}{l}\text { Bacteremia } \\
(n=21)\end{array}$ & SSTI $(n=9)$ & $\begin{array}{l}\text { MSK infection } \\
(n=11)\end{array}$ \\
\hline Age (years) (median, IQR) & $5(2.5,10.5)$ & $3.6(2.5,13)$ & $4.2(3.9,8.3)$ & $7.2(1.6,11.4)$ \\
\hline Male sex & $21(46 \%)^{\mathrm{a}}$ & $10(48 \%)$ & $4(44 \%)$ & $5(45 \%)$ \\
\hline \multicolumn{5}{|l|}{ Race/ethnicity } \\
\hline White & $37(80 \%)$ & $15(71 \%)$ & $7(77 \%)$ & $10(91 \%)$ \\
\hline Black & $7(15 \%)$ & $4(19 \%)$ & $2(22 \%)$ & $1(9 \%)$ \\
\hline Hispanic/Latino & $4(9 \%)$ & $2(10 \%)$ & 0 & $2(18 \%)$ \\
\hline \multicolumn{5}{|l|}{ Chronic disease } \\
\hline Heart disease & $2(4 \%)$ & $2(10 \%)$ & 0 & 0 \\
\hline Gastrointestinal & $7(15 \%)$ & $7(33 \%)$ & 0 & 0 \\
\hline Oncologic & $9(20 \%)$ & $9(43 \%)$ & 0 & 0 \\
\hline None & $21(46 \%)$ & $2(10 \%)$ & $9(100 \%)$ & $8(73 \%)$ \\
\hline ICU admission & $5(11 \%)$ & $2(10 \%)$ & 0 & $1(9 \%)$ \\
\hline Surgical procedure ${ }^{\mathrm{b}}$ & $16(34 \%)$ & $4(19 \%)$ & $3(33 \%)$ & $7(64 \%)$ \\
\hline $\begin{array}{l}\text { Baseline PCT }(\text { mean } \pm \text { SD) } \\
\quad(\text { median; IQR })\end{array}$ & $\begin{array}{l}6.41 \pm 13.62(0.76 \\
0.11,5.96)\end{array}$ & $\begin{array}{l}4.26 \pm 5.95(0.91 \\
0.4,5.9)\end{array}$ & $\begin{array}{l}1.73 \pm 3.7(0.27 \\
0.11,0.54)\end{array}$ & $\begin{array}{c}7.11 \pm 10.41(2.12 \\
0.09,10.98)\end{array}$ \\
\hline
\end{tabular}

$S S T I$ skin/soft tissue infection, $M S K$ musculoskeletal, $I C U$ intensive care unit, PCT procalcitonin, $S D$ standard deviation, $I Q R$ interquartile range

a Percent represents percent of participants in each column

b Participant underwent surgical procedure during the study period (on or after the day of enrollment)

musculoskeletal infection $(7.1 \mu \mathrm{g} / \mathrm{l}, n=11)$ were higher than other infection types. Median PCT levels declined faster than median CRP levels (Fig. 1). Of the 11 participants with musculoskeletal infection, 7 (64\%) underwent surgery during the study time period (3 participants on the day that the baseline PCT level was obtained and 4 participants on day 1). Procalcitonin kinetics followed a half-life of approximately $24 \mathrm{~h}$ in most patients (Fig. 2). Of the 39 patients who had PCT levels drawn at baseline and on day $1,8(21 \%)$ had a decrease of at least $50 \%$ in their PCT value on day 1 , and 14 of the 37 patients $(37.8 \%)$ who had PCT levels drawn at baseline and on day 2 had a decrease of at least $50 \%$ on day 2 . Restricting only to patients with initial PCT levels $>0.5 \mu \mathrm{g} / \mathrm{l}, 8$ of 25 patients (32\%) with PCT levels drawn on baseline and day 1 had at least 50\% decline in PCT on day 1 ,

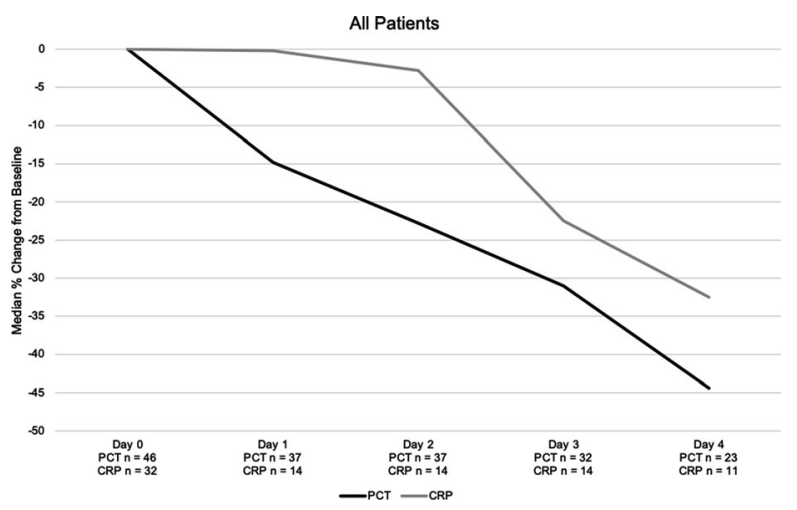

Fig. 1 Median percent change in biomarker levels during treatment. Black line indicates median percent change in procalcitonin levels from baseline, and gray line indicates median percent change in C-reactive protein levels from baseline over the study period. PCT procalcitonin, CRP C-reactive protein 

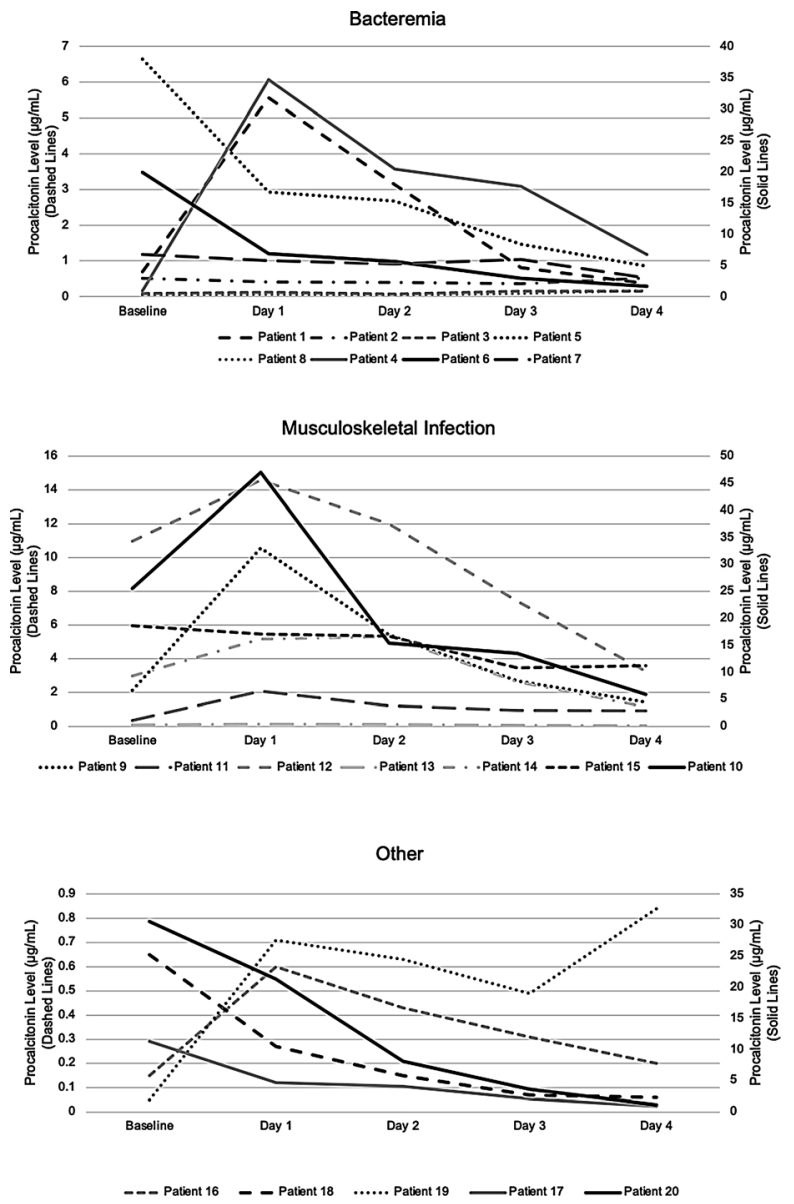

Fig. 2 Daily procalcitonin levels among 20 participants with levels measured per protocol. Each line represents a unique participant. "Other" diagnoses include skin/soft tissue infection $(n=2)$, urinary tract infection $(n=2)$ and meningitis $(n=1)$

and 12 of 24 patients (50\%) with PCT levels drawn on baseline and day 2 had a decline in PCT value of at least $50 \%$. There was variation in biomarker levels among participants with the same infection type, especially in participants with bacteremia, musculoskeletal infection and skin/soft tissue infection (Fig. 2 and Table 2). Most participants had declining PCT levels throughout the study, but some had stable or rising levels throughout the study duration. Most participants with stable or rising levels had low PCT levels $(<1 \mu \mathrm{g} / \mathrm{l})$ throughout the duration of the study (Fig. 2).

\section{DISCUSSION}

Although PCT is commonly used in the clinical setting to predict severe bacterial infection and to guide antibiotic de-escalation in both adults and children, the utility of PCT to predict response to antibiotic therapy is unclear, especially in children. Comparisons of serial PCT and CRP in this prospective cohort of hospitalized children with serious bacterial infections revealed that mean baseline PCT was high in all participants and highest for those with meningitis and musculoskeletal infections. PCT declined faster than CRP over the first $48 \mathrm{~h}$ of appropriate antibiotic treatment in all participants, including those with musculoskeletal infection who underwent surgery within the first $48 \mathrm{~h}$ of enrollment. Due to small sample size, we were not able to determine whether PCT declined faster than CRP in participants with bacteremia or skin/soft tissue infection.

If PCT normalizes faster than CRP, trending PCT instead of CRP could be cost saving in the long term because fewer tests would be needed. A recent study demonstrated that CRP outperformed PCT in diagnosing pediatric musculoskeletal infection, so measurement of PCT alone on initial evaluation may not be sufficient [14]. Another possible use for PCT is in reducing the duration of antibiotic courses for non-critical bacterial infections or facilitating earlier conversion from intravenous to oral antibiotics, although more research is needed on this topic before recommendations to support this clinical application can be made.

The decline in PCT levels that we noted among participants receiving adequate antibiotic therapy provides supporting evidence that PCT may add useful information to clinical prediction scores, especially for musculoskeletal infection. PCT is currently used, along with other laboratory and clinical characteristics, in the "step-by-step" approach, a prediction score that aids clinicians to safely avoid lumbar punctures in febrile infant evaluations [1]. Low PCT values have also been associated with reduced odds of ICU admission and shorter length of hospital stay compared with higher PCT concentrations in the Etiology of 
Table 2 Detailed information on organism and surgery date among 20 participants with procalcitonin levels measured per protocol

\begin{tabular}{llll}
\hline ID & Infection type & Organism & Surgery (yes or no), day of surgery \\
\hline 1 & Bacteremia & E. coli & No \\
2 & Bacteremia & E. coli & No \\
3 & Bacteremia & Enterococcus spp. & No \\
4 & Bacteremia & P. aeruginosa & No \\
5 & Bacteremia & E. coli & No \\
6 & Bacteremia & S. pneumoniae & No \\
7 & Bacteremia & S. aureus & Yes, day 4 \\
8 & MSKI & S. aureus & Yes, day 3 \\
9 & MSKI & Negative & Yes, day 1 \\
10 & MSKI & Negative & No \\
11 & MSKI & MSSA & No \\
12 & MSKI & MRSA & Yes, day 2 \\
13 & MSKI & MSSA & Yes, day 2 \\
14 & MSKI & H. influenzae & Yes, day 2 \\
15 & MSKI & MRSA & Yes, day 2 \\
16 & SSTI & MRSA & Yes, day 2 \\
17 & SSTI & S. pyogenes & Yes, day 2 \\
18 & UTI & E. coli & No \\
19 & UTI & Negative (culture was pre-treated with antibiotics) & Yes, day 2 \\
20 & Meningitis & S. pneumoniae & No \\
\hline
\end{tabular}

MSKI musculoskeletal infection, SSTI skin/soft tissue infection, UTI urinary tract infection

Pneumonia in the Community (EPIC) study, a multicenter study of 532 hospitalized children with pneumonia [24]. Recent prediction scores developed to identify complications of acute hematogenous osteomyelitis in children include biomarkers such as total leukocyte count, erythrocyte sedimentation rate (ESR) and CRP along with clinical characteristics $[25,26]$. The inclusion of single or serial PCT values could further strengthen these prediction scoring systems and warrants further research. A recent meta-analysis demonstrated only moderate diagnostic accuracy of PCT for diagnosis of bacterial pneumonia in children, highlighting the fact that PCT should not be used in isolation to diagnose bacterial infections in children [16].

This study has several limitations. It was a single-center study with a small sample size (increasing probability of a type II error and decreasing generalizability), and we had difficulty obtaining all five blood draws among enrolled participants. We enrolled participants with a variety of bacterial infections, which increases generalizability but also decreases the ability to draw meaningful conclusions for individual diagnoses. We could not evaluate the utility of PCT as a marker of treatment failure or poor clinical outcome as we did not have any 
participants whose infection did not respond to antibiotic therapy, and all had good outcomes. Not all patients enrolled had culture-confirmed bacterial diagnoses, so it is possible that these patients' elevated PCT levels may have been due to a cause other than bacterial infection. Finally, CRP levels were drawn as part of routine clinical care and were not directed by the study protocol, so corresponding levels were only available for $32 / 46$ (70\%) participants at baseline and 11/46 (24\%) on day 4. Participants with CRP levels drawn on day 4 were also more likely to be sicker than those without CRP levels as they had longer hospital stays and clinicians chose to monitor their inflammatory biomarkers. We expected all participants with bacteremia to have high PCT levels and were surprised to see that some did not. It is possible that participants with lower levels had low-level colonization of their central lines without systemic bacteremia. Despite these limitations, this study is one of only a few evaluating serial PCT levels among pediatric patients and provides valuable insight into the kinetics of PCT in children receiving effective therapy for noncritical, acute bacterial infection.

\section{CONCLUSION}

PCT is a promising biomarker to follow every $24-48 \mathrm{~h}$ to monitor response to antibiotic therapy and appears to decline more quickly than CRP in response to effective therapy. More research is needed to determine the optimal monitoring frequency for PCT and to understand its utility as a prognostic biomarker in children with a variety of infections.

\section{ACKNOWLEDGEMENTS}

Funding. This work was supported by an investigator-initiated research agreement through bioMérieux and by the National Institute of Allergy and Infectious Diseases Childhood Infection Research Program (ChIRP), National Institute of Health (grant no. 1T32AI095202-07). This content is solely the responsibility of the authors and does not necessarily represent the official views of the National Institutes of Health. S.K. received grant support from the Centers for Disease Control and Prevention as a recipient of the Leadership in Epidemiology, Antimicrobial Stewardship and Public Health (LEAP) fellowship, sponsored by the Society for Healthcare Epidemiology of America (SHEA), Infectious Diseases Society of America (IDSA) and Pediatric Infectious Diseases Society (PIDS). We are grateful to bioMérieux for providing a PCT testing platform and funds for the journal's Rapid Service Fees. The company was not involved in study design, conduct, data analysis, manuscript preparation or publication.

Authorship. All named authors meet the International Committee of Medical Journal Editors (ICMJE) criteria for authorship for this article, take responsibility for the integrity of the work as a whole, and have given their approval for this version to be published.

Disclosures. Sophie E. Katz, Jennifer Crook, Rendie McHenry, Andras Szeles, Natasha Halasa and Ritu Banerjee have no conflicts of interest relevant to this article to disclose.

Compliance with Ethics Guidelines. This study was approved by the Vanderbilt University institutional review board. The study was performed in accordance with the Helsinki Declaration of 1964 and its later amendments. All subjects provided informed consent to participate in the study.

Data Availability. The datasets generated during and/or analyzed during the current study are available from the corresponding author on reasonable request.

Open Access. This article is licensed under a Creative Commons Attribution-NonCommercial 4.0 International License, which permits any non-commercial use, sharing, adaptation, distribution and reproduction in any medium or format, as long as you give appropriate credit to the original author(s) and the source, provide a link to the Creative Commons licence, and 
indicate if changes were made. The images or other third party material in this article are included in the article's Creative Commons licence, unless indicated otherwise in a credit line to the material. If material is not included in the article's Creative Commons licence and your intended use is not permitted by statutory regulation or exceeds the permitted use, you will need to obtain permission directly from the copyright holder. To view a copy of this licence, visit http://creativecommons.org/licenses/by$\mathrm{nc} / 4.0 /$.

\section{REFERENCES}

1. Gomez B, Mintegi S, Bressan S, Dalt LD, Gervaix A, Lacroix L, et al. Validation of the "step-by-step" approach in the management of young febrile infants. Pediatrics (Internet). 2016;138(2). https:// pediatrics.aappublications.org/content/138/2/ e20154381. Accessed 31 Mar 2020.

2. Kuppermann N, Dayan PS, Levine DA, Vitale M, Tzimenatos L, Tunik MG, et al. A clinical prediction rule to identify febrile infants 60 days and younger at low risk for serious bacterial infections. JAMA Pediatr. 2019;173(4):342-51.

3. de Jong E, van Oers JA, Beishuizen A, Vos P, Vermeijden WJ, Haas LE, et al. Efficacy and safety of procalcitonin guidance in reducing the duration of antibiotic treatment in critically ill patients: a randomised, controlled, open-label trial. Lancet Infect Dis. 2016;16(7):819-27.

4. Christ-Crain M, Jaccard-Stolz D, Bingisser R, Gencay MM, Huber PR, Tamm M, et al. Effect of procalcitonin-guided treatment on antibiotic use and outcome in lower respiratory tract infections: cluster-randomised, single-blinded intervention trial. Lancet. 2004;363(9409):600-7.

5. Downes KJ, Fitzgerald JC, Schriver E, Boge CLK, Russo ME, Weiss SL, et al. Implementation of a pragmatic biomarker-driven algorithm to guide antibiotic use in the pediatric intensive care unit: the Optimizing Antibiotic Strategies in Sepsis (OASIS) II study. J Pediatric Infect Dis Soc. 2020;9(1):36-43.

6. Nobre V, Harbarth S, Graf J-D, Rohner P, Pugin J. Use of procalcitonin to shorten antibiotic treatment duration in septic patients: a randomized trial. Am J Respir Crit Care Med. 2008;177(5):498-505.
7. Schuetz P, Birkhahn R, Sherwin R, Jones AE, Singer A, Kline JA, et al. Serial procalcitonin predicts mortality in severe sepsis patients: results from the Multicenter Procalcitonin MOnitoring SEpsis (MOSES) study. Crit Care Med. 2017;45(5):781-9.

8. Schuetz P, Briel M, Christ-Crain M, Stolz D, Bouadma L, Wolff M, et al. Procalcitonin to guide initiation and duration of antibiotic treatment in acute respiratory infections: an individual patient data meta-analysis. Clin Infect Dis. 2012;55(5): 651-62.

9. Dandona P, Nix D, Wilson MF, Aljada A, Love J, Assicot $\mathrm{M}$, et al. Procalcitonin increase after endotoxin injection in normal subjects. J Clin Endocrinol Metab. 1994;79(6):1605-8.

10. Brunkhorst FM, Heinz U, Forycki ZF. Kinetics of procalcitonin in iatrogenic sepsis. Intensive Care Med. 1998;24(8):888-9.

11. Azzini AM, Dorizzi RM, Sette P, Vecchi M, Coledan I, Righi E, et al. A 2020 review on the role of procalcitonin in different clinical settings: an update conducted with the tools of the evidence based laboratory medicine. Ann Transl Med [Internet]. 2020;8(9). https://www.ncbi.nlm.nih.gov/pmc/ articles/PMC7290560/. Accessed 1 Oct 2020.

12. Metlay JP, Waterer GW, Long AC, Anzueto A, Brozek J, Crothers K, et al. Diagnosis and treatment of adults with community-acquired pneumonia. An official clinical practice guideline of the American Thoracic Society and Infectious Diseases Society of America. Am J Respir Crit Care Med. 2019;200(7): e45-67.

13. Rhodes A, Evans LE, Alhazzani W, Levy MM, Antonelli M, Ferrer R, et al. Surviving sepsis campaign: International Guidelines for Management of Sepsis and Septic Shock: 2016. Crit Care Med. 2017;45(3):486-552.

14. McMichael BS, Nickel AJ, Christensen EW, Frenn $\mathrm{KA}$, Truong WH, Laine JC, et al. Discriminative accuracy of procalcitonin and traditional biomarkers in pediatric acute musculoskeletal infection. Pediatr Emerg Care. 2020.

15. Florin TA, Ambroggio L, Brokamp C, Zhang Y, Rattan M, Crotty E, et al. Biomarkers and disease severity in children with community-acquired pneumonia. Pediatrics. 2020;145(6).

16. Tsou P-Y, Rafael J, Ma Y-K, Wang Y-H, Raj S, Encalada $S$, et al. Diagnostic accuracy of procalcitonin for bacterial pneumonia in children-a systematic review and meta-analysis. Infect Dis. 2020;52(10):683-97. 
17. Damman J, Arias P, Kerner J, Zhang K-Y, Dehghan M, Krishnan G, et al. Procalcitonin as a predictive marker for bacteremia in children with a central line and fever. Hosp Pediatr. 2019;9(6):434-9.

18. Shaikh N, Martin JM, Hoberman A, Skae M, Milkovich L, Nowalk A, et al. Host and bacterial markers that differ in children with cystitis and pyelonephritis. J Pediatr. 2019;209(146-153):e1.

19. Meisner M. Update on procalcitonin measurements. Ann Lab Med. 2014;34(4):263-73.

20. Boussekey N, Leroy O, Alfandari S, Devos P, Georges $\mathrm{H}$, Guery B. Procalcitonin kinetics in the prognosis of severe community-acquired pneumonia. Intensive Care Med. 2006;32(3):469-72.

21. Shi Y, Xu Y, Rui X, Zhang H, Wang Y, Du W. Procalcitonin kinetics and nosocomial pneumonia in older patients. Respir Care. 2014;59(8):1258-66.

22. Jenkins TC, Haukoos JS, Cotton E, Weitzenkamp D, Frank DN, Burman WJ. Time course of C-reactive protein and procalcitonin levels during the treatment of acute bacterial skin infections. Open Forum Infect Dis. 2018;5(3):ofy029.
23. Bobillo S, Rodríguez-Fanjul J, Solé A, Moreno J, Balaguer M, Esteban E, et al. Kinetics of procalcitonin in pediatric patients on extracorporeal membrane oxygenation. Biomark Insights. 2018;13: 117727191775190.

24. Stockmann C, Ampofo K, Killpack J, Williams DJ, Edwards KM, Grijalva CG, et al. Procalcitonin accurately identifies hospitalized children with low risk of bacterial community-acquired pneumonia. J Pediatric Infect Dis Soc. 2018;7(1):46-53.

25. Alhinai Z, Elahi M, Park S, Foo B, Lee B, Chapin K, et al. Prediction of adverse outcomes in pediatric acute hematogenous osteomyelitis. Clin Infect Dis [Internet]. https://academic.oup.com/cid/advancearticle/doi/10.1093/cid/ciaa211/5780104. Accessed 31 Mar 2020.

26. Copley LAB, Barton T, Garcia C, Sun D, GaviriaAgudelo C, Gheen WT, et al. A proposed scoring system for assessment of severity of illness in pediatric acute hematogenous osteomyelitis using objective clinical and laboratory findings. Pediatr Infect Dis J. 2014;33(1):35-41. 